\title{
Medicina di Laboratorio centrata sul paziente
}

\section{Patient-centered Laboratory Medicine}

\author{
Piero Cappelletti ${ }^{1}{ }_{\mathbb{C}}$
}

Ricevuto: 5 dicembre 2016 / Accettato: 7 dicembre 2016

(c) Società Italiana di Patologia Clinica e Medicina di Laboratorio 2016

Riassunto Al passaggio del millennio la Medicina occidentale si è interrogata sui successi e fallimenti della sua efficacia clinica e della sua organizzazione, individuando nell'effetto "silo" un tema cruciale dell'assistenza sanitaria e della salute dei pazienti. Per "centralità del paziente" s'intende rispetto per le preferenze e i valori del paziente, conforto fisico, sostegno emotivo, integrazione, coordinazione e continuità delle cure, coinvolgimento della famiglia e degli amici, informazione, comunicazione ed educazione dei pazienti al fine di comprendere e collaborare alle decisioni inerenti alla loro salute. Il concetto della centralità del paziente in Medicina di Laboratorio è stato declinato a diversi livelli, con riguardo alla salvaguardia delle persone, alla costruzione di un' organizzazione focalizzata sul paziente e alla fornitura di un servizio di alta qualità. Solo una visione globale del lavoro all'interfaccia, curato da un'equipe multitasking e rivolto a una rete di utilizzatori di cui il paziente è il fulcro, è in grado di sorreggere adeguatamente la sfida di una Medicina di Laboratorio veramente centrata sul paziente.

Parole chiave Medicina di Laboratorio $\cdot$ Centralità del paziente $\cdot$ Sicurezza del paziente $\cdot$ Point-of-care testing $(\mathrm{POCT}) \cdot$ Total testing process $(\mathrm{TTP})$

Summary At the beginning of the new millennium, Western Medicine face to successes and failures of its effectiveness and organization identified the "silos" effect as a main issue of the health care systems. The centeredness of the patient became crucial. Patient-centered care covers some domains including respect for patients' preferences and values, physical comfort, emotional support, integration and coordination, continuity of care, involvement of family and friends,

$凶$ P. Cappelletti pie.cappelletti@gmail.com

1 SIPMeL, Castelfranco Veneto (TV), Italia and the provision of information, communication and education to enable patients to understand and make informed decisions about their care. For Laboratory Medicine, the "patient-centered" concept is applicable to the issues of patients' safety and rights, of a laboratory organization built around the patient, and of a Total Testing Process focused on the patients' needs and preferences. Nevertheless, only a comprehensive vision of the clinic-laboratory interface work by a multitasking team and focused on a network of patientcentered clinical users allows facing a real Patient Centered Laboratory Medicine.

Keywords Laboratory Medicine P Patient-centered Patient safety $\cdot$ Point-of-care testing $(P O C T) \cdot$ Total testing process (TTP)

\section{Introduzione}

Al passaggio del millennio la Medicina occidentale si è interrogata sui successi e fallimenti della sua efficacia clinica e della sua organizzazione, individuando alla luce del dibattito sulla "qualità" gli obiettivi di una nuova stagione dell' assistenza sanitaria e della salute dei pazienti.

Infatti, nonostante l'oggettivo miglioramento delle cure, i pazienti lamentano frequenti fallimenti nel risk assessment personalizzato e nella comunicazione nel team e con il paziente, disallineamenti tra ambiti di cura, obiettivi, rischi e uso della tecnologia, insufficienti rivalutazioni iterative degli obiettivi di cura e sperimentano il cosiddetto "effetto silo" caratterizzato da frammentazione, scarsa integrazione, sovrapposizione e carenze nei diversi ambiti di cura, che induce altresì diffuse conseguenze psicosociali negative (nightmare of healthcare) e circa il $30 \%$ di costi che potrebbe essere speso più efficacemente [1]. 
Nel mondo americano, l'Institute of Medicine (IOM), nel suo rapporto Crossing the Quality Chasm [1] del 2001, identifica i quattro principali motivi delle difficoltà sanitarie sopra ricordate (la crescente complessità della scienza e della tecnologia, l'aumento delle malattie croniche, un'organizzazione sanitaria inadeguata e i limiti nel pieno utilizzo della rivoluzione informatica) e i sei obiettivi di un sistema sanitario adeguato alle sfide contemporanee e spesso dimenticati nell'ambiente, nei processi e nelle strutture destinate all'assistenza. La Medicina e la sua organizzazione devono essere sicure, efficaci, centrate sul paziente, tempestive, efficienti ed eque.

Nel mondo anglosassone, il grande sforzo riformatore della Modernizzazione [2] del NHS britannico poggia su principi simili, seppure declinati con differente priorità: il sistema sanitario deve essere centrato sul paziente, valorizzante lo staff, equo, efficace, integrato, efficiente e appropriato. In Canada [3] i principi riconosciuti della qualità sanitaria sono l'accessibilità, l'efficacia, la sicurezza, la centralità del paziente, l'equità, l'efficienza, la disponibilità di risorse adeguate, l'integrazione e la focalizzazione sulla salute delle popolazioni. In Australia [4] il sistema sanitario è costruito centrato sulla popolazione e la famiglia, equo, a responsabilità condivisa, volto alla prevenzione e al benessere, globale e di valore, nel senso di cure appropriate, tempestive ed efficaci basate sulla migliore evidenza disponibile e con l'obiettivo della più alta qualità possibile. Si notino le assonanze, in particolare per quanto riguarda il tema della centralità del paziente, e le differenze, figlie delle diverse storie e dei contesti sociali dei Paesi citati.

In Italia [5] i principi fondamentali del sistema sanitario sono l'universalità, l'uguaglianza e l'equità e quelli organizzativi la centralità della persona, la responsabilità pubblica della tutela della salute, la collaborazione tra i livelli di governo del Servizio sanitario nazionale (SSN), la valorizzazione delle professioni e l'integrazione sociosanitaria.

\section{Centralità del paziente}

Ancora nel 1993 una ricerca [6] della Harvard School of Medicine e del Piker Institute definì che cosa si debba intendere per centralità del paziente, identificando otto aree specifiche: rispetto per le preferenze e i valori del paziente, conforto fisico, sostegno emotivo, integrazione, coordinazione e continuità delle cure, coinvolgimento della famiglia e degli amici, informazione, comunicazione ed educazione dei pazienti al fine di comprendere e collaborare alle decisioni inerenti alla loro salute. Benché l'Organizzazione Mondiale della Sanità (OMS) preferisca il termine "responsiveness" per identificare la capacità di un'organizzazione sanitaria di rispondere alle aspettative del paziente in ordine a rispetto, preferenze, comunicazione e attese, il termine "patient centered" è il più diffuso in letteratura [7]. Talora si utilizza il termine "consumer centered" per evitare la passività insita nel termine "paziente" e allineare il concetto a quello di "cliente" con i propri diritti, preferenze e valutazione della qualità. Talaltra è preferito il termine "person-centered", specialmente nella Medicina territoriale e della terza età, per sottolineare le caratteristiche di condivisione della gestione delle malattie croniche da parte del paziente. Non sfuggirà il valore ideologico che può assumere la traduzione italiana. Infatti, in Italia [5] si parla di "centralità della persona" che si declina in una serie di diritti esercitabili da parte dei singoli cittadini e che rappresentano dei doveri per tutti gli operatori sanitari, dal medico a chi programma l'assistenza territoriale. I diritti principali sono la libertà di scelta del luogo di cura, il diritto a essere informato sulla malattia e sulla terapia e a opporsi o dare il consenso, il diritto di "essere preso in carico" dal medico o dall'équipe sanitaria durante tutto il percorso terapeutico, il diritto alla riservatezza e il dovere della programmazione sanitaria di anteporre la tutela della salute dei cittadini a tutte le scelte, compatibilmente alle risorse economiche disponibili.

Il concetto di centralità del paziente, inoltre, ha un focus specifico sul personale, le cui abilità, inclinazioni ed esperienza sono cruciali per una cura attenta ai principi suesposti. E d'altro canto emergono aspetti specifici delle organizzazioni sanitarie che caratterizzano tale prospettiva, in particolare per quanto attiene uno stile di consultazione attento al paziente, l'addestramento del personale a una comunicazione adeguata, feedback continuo dell'esperienza dei pazienti e pubblicità dei dati di performance [7]. È importante sottolineare l'impatto di un tale approccio sulla gestione delle malattie croniche che oggi rappresentano il grande problema sanitario del mondo e la connessione con le proposte organizzative per fronteggiarlo. Una delle più accettate risposte alla complessità dei problemi posti dalle malattie croniche è il Chronic Care Model (CCM) [8]. Il CCM è un quadro di riferimento evidence-based per i cambiamenti sanitari, in particolar modo nella Medicina territoriale, che si basa sulla fruttuosa interazione tra un paziente informato attivo e un team sanitario preparato e proattivo. Le principali componenti sono il self-management come parte della collaborazione del paziente, nuovi modi di praticare la Medicina (proattiva, integrata e prossima), supporti di evidenze (EBM, linee guida) e un sistema informativo clinico, sempre accessibile e tempestivo. L'adattamento internazionale di CCM è il WHO Innovative Care for Chronic Conditions (ICCC) Framework [9]. A livello micro la diade paziente-team diventa una triade: (1) paziente (informato, motivato, preparato) (2) team (continuità, coordinazione, qualità e organizzazione, più ITC e self management) (3) comunità (consapevolezza, incoraggiamento, risorse, servizi complementari). ICCC sottolinea a livello meso il ruolo della comunità organizzata e a livello macro l'importanza di partner e stakeholder e di politiche integrate così come del finanziamento e dell'advocacy. 
Infine, esistono diverse evidenze di letteratura, seppure non di livello definitivo, che confermano gli effetti positivi di un'attitudine centrata sul paziente dell'organizzazione sanitaria, con riferimento a una maggiore soddisfazione dei pazienti e del personale, migliori esiti primari (mortalità) e secondari (dimissioni e riammissioni, lunghezza della degenza), diminuzione degli errori e percezione dello staff di un cambiamento positivo nell' assistenza [7]. Così che si può concludere che il concetto della centralità del paziente non è solo filosofico, ma anche del tutto pratico [10] e comprende come elemento essenziale l'accountability.

\section{Medicina di Laboratorio centrata sul paziente}

Il concetto della centralità del paziente può essere applicato alla Medicina di Laboratorio a diversi livelli con riferimento ai bisogni, ai diritti, alle preferenze e ai valori dei pazienti. In particolare la centralità del paziente in Medicina di Laboratorio è stata declinata riguardo alla salvaguardia delle persone, alla costruzione di un'organizzazione focalizzata al paziente e alla fornitura di un servizio di alta qualità.

Per il primo punto fu di fondamentale importanza l'appendice allo Standard di Certificazione ISO 15189:2003 [11], denominata Ethics in Laboratory Medicine, che descrive le specifiche non tecniche, ma appunto etiche, della raccolta delle informazioni (trasparenza e confidenzialità) e dei campioni (consenso esplicito e implicito), dell'esecuzione dell'esame (secondo lo stato dell'arte, evidencebased), della refertazione (soggetto abilitato a riceverlo), dell'interpretazione (responsabilità del laboratorio, consulenza), della conservazione e tracciabilità della documentazione e della sua accessibilità (soggetto abilitato), dell'utilizzo di campioni analitici per esami diversi da quelli originariamente richiesti (anonimizzazione e pooling) e del divieto di accordi economici a discapito dei pazienti. Nella parte di generalità, lo standard stressa il punto che per la Medicina di Laboratorio nella maggior parte dei casi il "patto di fiducia" medico-paziente non è diretto ed esplicito, ma che, comunque, nel patto Laboratorio-Richiedente l'interesse e il benessere del paziente devono essere sempre previlegiati. Negli stessi anni, sulla base delle indagini che dimostrano la difettosità dei sistemi sanitari e del laboratorio in particolare [12], la patient-safety diviene un aspetto importante degli standard di accreditamento e un obiettivo principale del miglioramento qualitativo dei sistemi sanitari. L'elaborazione italiana di SIMeL [13] di questi concetti si configura nei termini dell'evoluzione del concetto che "patient safety is not enough" in una big picture di cerchi concentrici di sempre più ampia salvaguardia dei pazienti: sicurezza del risultato come qualità analitica, sicurezza del processo come qualità del TTP, sicurezza dell'esito come appropriatezza ed effectiveness, sicurezza del cittadino come ruolo sociale della Medicina di Laboratorio. In Italia in quegli anni vi è una sensibilità di associazioni (CittadinanzAttiva) e Società scientifiche (SIMeL) per i diritti dei cittadini-pazienti. Nel 2002 viene stesa e approvata la Carta europea dei diritti del malato [14]. In Italia ricerche in tal senso sono state operate a più riprese da CittadinanzAttiva (2001; 2004; 2006). Nel 2004-2006 un' approfondita indagine su "Diritti dei cittadini e laboratorio analisi" [15] esamina il rispetto dei quattordici diritti fondamentali (misure preventive, accesso, informazione, consenso, libera scelta, tempo del paziente, privacy e confidenzialità, standard di qualità, sicurezza, innovazione, evitare sofferenze, trattamento personalizzato, reclamo, risarcimento) con risultati soddisfacenti, seppure a macchia di leopardo, sul territorio nazionale e con molti miglioramenti possibili nell'informazione, nel consenso e nella documentazione-tracciabilità dei dati. Oggi questi punti rappresentano la base dei Laboratori ad alto valore aggiunto, che determina anche cambiamenti competenziali [16].

Un secondo punto di vista è quello della costruzione di organizzazioni di laboratorio incentrate sul paziente, in particolare l'organizzazione del total testing process (TTP) in termini di "point of need" (ospedale, territorio, home, self), "timeliness" (organizzazione e automazione intorno alle necessità del paziente) e presa in carico del paziente nelle reti o nei Percorsi Diagnostico-Terapeutici Assistenziali (PDTA) [17]. Il modello "integrato" di Laboratorio è caratterizzato dall'assenza di pareti, dall'integrazione di diverse modalità di declinazione della Medicina di Laboratorio, dall'amplificazione dell'interfaccia clinica-laboratorio su tutta la rete di rapporti clinici, dal nuovo ruolo dei professionisti di laboratorio nel disegnarlo a misura di paziente, dall'implementazione e valutazione di nuove linee guida e mappe di cura, da un'équipe multidisciplinare, da un sistema strumentale multitasking mirato alla qualità e alla tempestività e dall'assunzione di un ruolo centrale dei sistemi di information and communication technology (ICT). Il modello di laboratorio "virtuale" centrato sul paziente, proposto inizialmente da Richardson [18] nel 1999, incorpora un primissimo livello di point-of-care testing (POCT), connesso a un secondo livello più esterno di Laboratori a risposta rapida per le informazioni diagnostiche che il POCT non può dare, a un terzo livello di Laboratori core o multidisciplinari che gestiscono la routine e la risposta della maggior parte dei campioni, a un quarto livello di Laboratori specialistici a integrazione dei Laboratori core e infine a un ultimo livello di reference laboratories per i test esoterici o per expertise non comuni [17]. In particolare, clinici e stakeholder guardano al POCT come uno strumento in grado di aiutare il raggiungimento del doppio obiettivo di migliorare la qualità delle cure e di tagliare i costi, attraverso quattro vie: consentire trattamenti più rapidi, facilitare l'aderenza ai protocolli, adottare soluzioni tecnologiche a minor costo e ampliare la connettività 
wireless. Queste quattro aree resteranno punti chiave dell'attività ospedaliera, ma il contributo del POCT sarà non tanto sull'innovazione tecnologica, che pure è molto spiccata nella tele-diagnostica, quanto nei servizi di supporto, nella produzione e condivisione di dati informatici, nei programmi educativi al livello del singolo paziente e nel ruolo di catalizzatore di nuovi modelli sanitari focalizzati alla continuità delle cure [19].

In terzo luogo aspetti principali della professione coinvolti dal tema della centralità del paziente e determinanti per la qualità del servizio sono l'accessibilità in termini di fase preanalitica [20] (preparazione ai test; prelievo; ambulatori specialistici; selezione dei test), la risposta al quesito clinico [21] come organizzazione e comunicazione dei risultat, informazione/formazione all'interpretazione e come consulenza diretta al paziente $[22,23]$ e la medicina di laboratorio " $4 P s$ " con i nuovi test e/o i nuovi livelli diagnostici della Medicina di Laboratorio personalizzata [24] e/o di precisione [25]. Ciascuno di essi andrebbe ulteriormente approfondito. Tuttavia, qui va almeno messo in luce come questi temi "storici" per la Medicina di Laboratorio acquistino un significato più complesso e non da tutti accettato se riferiti direttamente al paziente. Nel 2005, nel paragrafo "The consumer, the physician, and the laboratory" della relazione congressuale [21], SIMeL affrontava il tema del rapporto con il paziente, che considerava centrale, anche se non dimenticava il rapporto con il medico curante e in particolare con il medico di medicina generale. Anni dopo, una revisione del ciclo di Lundberg [26] introduceva il paziente ma solo in rapporto con il clinico e dedicava un intero paragrafo all'impatto negativo del contatto diretto con il paziente da parte del Laboratorio. Più recentemente, si è evidenziato come il TTP debba essere declinato intorno al paziente per ottenere risultati adeguati in termini di appropriatezza [27], ma anche qui il rapporto con il paziente è tenuto solo dal suo medico curante.

Solo una visione globale del lavoro all'interfaccia, curato da un'equipe multitasking e rivolto a una rete di utilizzatori di cui il paziente è il fulcro, è in grado di sorreggere adeguatamente la sfida di una Medicina di Laboratorio veramente centrata sul paziente [28]. E almeno su questo siamo tutti (più o meno) d'accordo [29].

\section{Conflitti di interesse Nessuno.}

Studi condotti su esseri umani e animali Per questo tipo di studio non è richiesto l'inserimento di alcuna dichiarazione relativa agli studi effettuati su esseri umani e animali.

\section{Bibliografia}

1. https://www.nationalacademies.org/hmd/ /media/Files/Report $\%$ 20Files/2001/Crossing-the-Quality-Chasm/Quality\%20Chasm $\% 202001 \% 20 \% 20$ report\%20brief.pdf (Accesso 30 novembre 2016)
2. https://www.gov.uk/government/uploads/system/uploads/ attachment_data/file/266003/newnhs.pdf (Accesso 30 novembre 2016)

3. https://www.oha.com/CurrentIssues/keyinitiatives/ PhysicianandProfessionalIssues/QPSGT/Documents/ Module\%206\%20-\%206.1.pdf (Accesso 30 novembre 2016)

4. http://www.health.gov.au/internet/nhhrc/publishing.nsf/Content/ principles-lp (Accesso 30 novembre 2016)

5. http://www.salute.gov.it/portale/salute/p1_5.jsp?id=21\&area=Il_ Ssn (Accesso 30 novembre 2016)

6. Gerteis M, Edgman-Levitan S, Daley J et al (1993) Through the patient's eyes: understanding and promoting patient-centered care. Jossey-Bass: San Francisco, CA

7. https://www.safetyandquality.gov.au/wp-content/uploads/2012/ 01/PCCC-DiscussPaper.pdf (Accesso 30 novembre 2016)

8. Wagner EH, Davis C, Schaefer J et al (1999) A survey of leading chronic disease management programs: are they consistent with literature? Manag Care Q 7:56-66

9. Epping-Jordan JE, Pruitt SD, Bengoa R et al (2004) Improving the quality of health care for chronic conditions. Qual Saf Health Care 13:299-305

10. Charmel PA, Frampton SB (2008) Building the business case for patient-centred care. Healthc Financ Manage 62:80-85

11. ISO 15189:2003 Medical laboratories-Particular requirements for quality and competence. Annex C: Ethics in Laboratory Medicine. http://asse.edu.iq/share/lab7.pdf (Accesso 30 novembre 2016)

12. Bonini P, Plebani M, Ceriotti F et al (2002) Errors in laboratory medicine. Clin Chem 48:691-698

13. Cappelletti P (2006) La Sicurezza del Paziente e la Medicina di Laboratorio. RIMeL/IJLaM 2(S1):17-35

14. Terzi A, Moccia F, Terzi E (2006) La sicurezza del paziente e la Medicina di Laboratorio. Il punto di vista dei cittadini. RIMeL/IJLaM 2(S1):42-48

15. http://www.cittadinanzattiva.it/files/corporate/europa/carta/carta_ europea_diritti_malato.pdf (Accesso 30 novembre 2016)

16. Morris S, Otto CN, Golemboski K (2013) Improving patient safety and healthcare quality in the 21 st century-competencies required of future medical laboratory science practitioners. Clin Lab Sci 26:200-204

17. Morandini M (2012) Scenari e ruoli della Medicina di Laboratorio al tempo della crisi: pianificazione, organizzazione e gestione. Riv Ital Med Lab 8:196-207

18. Richardson H (1999) Laboratory medicine in Ontario: its downsizing and the consequence on quality. Clin Chim Acta 290:57-72

19. Coyle MC (2009) Focusing on the Continuum of Care: How point-of-care testing solutions can help hospitals reduce costs and improve patient care. Point of Care 8:151-153

20. Cappelletti P (2004) Un patto per la modernizzazione e la umanizzazione della Medicina di Laboratorio in Italia. Riv Med Lab-JLM $5: 252-254$

21. Cappelletti P (2005) La risposta della Medicina di Laboratorio al quesito clinico. RIMeL/IJLaM 1(S1):15-23

22. Dorizzi RM (2006) Consulting: il supporto all'interpretazione. RIMeL/IJLaM 2:68-84

23. Cenci AM (2012) Scenari e ruoli della Medicina di Laboratorio al tempo della crisi: consulenza clinica. Riv Ital Med Lab 8:208-215

24. Cappelletti P (2009) La medicina personalizzata fra ricerca e pratica clinica: il ruolo della medicina di laboratorio. RIMeL/IJLaM 5(S1):26-32

25. Cappelletti P (2016) Medicina di Precisione e Medicina di Laboratorio. Riv Ital Med Lab 12:129-133 
26. Plebani M, Laposata M, Lundberg GD (2011) The brain-to-brain loop concept for laboratory testing 40 years after its introduction. Am J Clin Pathol 136:829-833

27. Freedman DB (2015) Towards better test utilization—strategies to improve physician ordering and their impact on patient outcomes. EJIFFC 26:15-30
28. Cappelletti P (2012) Scenari e ruoli della Medicina di Laboratorio ai tempi della crisi: da Modena a Salerno ed oltre. Riv Ital Med Lab 8:183-189

29. Hallworth MJ, Epner PL, Ebert C et al (2015) Current evidence and future perspectives on the effective practice of patient-centered laboratory medicine. Clin Chem 61:589-599 